\title{
Spectral Description of the Dissipation Mechanism for Wind Waves. Eddy Viscosity Model
}

\author{
Vladislav G. Polnikov
}

A.M. Obukhov Institute of Atmospheric Physics of Russian Academy of Sciences, Moscow, 117018, Russia

\begin{abstract}
The objective of this paper is to perform a theoretically justified parameterization of the dissipation function for wind waves in the spectral form. To solve the problem, the similarity method was used: similarity statements are formulated; dimensionless characteristics of the system are introduced; and general phenomenological representation of the dissipation function for wind waves $D I S(S)$ is constructed. The only additional assumption is that this presentation is valid in a local approximation in the wave spectrum $S$. Physical analysis of the general representation allows us to reduce the dissipation function to the form $D I S(S) \propto S^{2}$. The further systematic formulation of the final representation of phenomenological function $D I S(S)$ is performed by joining numerous previous results of the author. The physical meaning of the accepted parameterization parameters is analysed, and the correspondence of the parameterization with the new experimental facts found in this field for the last 5-10 years is shown. The previous numerical results are presented that illustrate a successful application of the constructed dissipation function. A regular theoretical justification of the revealed phenomenological representation of $D I S(S)$ is based on the well-known Hasselmann's approach[1]. This theory was modified by the system of postulates resulting in the dissipation mechanism realized due to turbulence in the upper water layer. Physically it means the eddy viscosity mechanism of wind wave dissipation. The final theoretical representation of the dissipation function corresponds to the phenomenological formulation: $D I S(S) \propto S^{2}$. This fact provides the physical justification of the phenomenological approach proposed.
\end{abstract}

Keywords Wind-wave, Spectra, Numerical Model, Source Function, Evolution Mechanism, Wind-wave Dissipation, Turbulence

\section{Introduction and Pose of the Problem}

Wind-sea is a stochastic hydrodynamic phenomenon taking place at the air-sea interface, in vicinity of which shear currents, waves, and turbulent motions take place. This multi-scale feature of motions provides for reasonable difficulties in solving the problem of wind wave dynamics description. Nevertheless, for a century and a half history of studying a physics of wind waves, a lot of points have gotten their solutions.

In particular, it is well established[2,3] that on the scales of hundreds of dominant wave lengths and periods, description of stochastic and non-stationary wave field is realized in terms of two-dimensional energy spectrum $S(\sigma, \theta ; \mathbf{x}, t) \equiv S$, spread in space $\mathbf{x}$ and time $t$ (here $\sigma$ and $\theta$ is the frequency and propagation direction of wave component, respectively, corresponding to the wave vector $\mathbf{k}(\sigma, \theta)$ ). In the case of deep water and neglecting a surface currents impact, the wave spectrum evolution is described by the following transport

* Corresponding author:

polnikov@mail.ru (Vladislav G. Polnikov)

Published online at http://journal.sapub.org/ms

Copyright (C) 2012 Scientific \& Academic Publishing. All Rights Reserved equation $^{1}$

$$
\frac{d S}{d t}=F \equiv N L+I N-D I S
$$

The left-hand side of (1.1) defines mathematics of a windsea model, which is not discussed here. But the left-hand side, called as source function (SF) $F$, contains physics of a model. In the frame of a certain series of assumptions, it is used to distinguish three main constituents of SF - three parts of the united evolution mechanism for wind waves:

- The rate of conservative nonlinear energy transfer through a wave spectrum, $N L$, ("nonlinear-term");

- The rate of energy transfer from wind to waves, IN, ("input-term");

- The rate of wave energy loss, DIS, ("dissipationterm").

A physical sense of equation (1.1) is evident: it is the energy conservation law applied to each of wave spectral components. Therefore, in principle, it can be postulated and written as an initial phenomenological equation. Nevertheless, a certain procedure exists, allowing derivation of this equation form a system of the basic hydrodynamic equations and specification a spectral representation for each term in

\footnotetext{
${ }^{1}$ More general representation of evolution equation (1.1) is not discussed here due to brevity description of well-known statements.
} 
the right-hand side of (1.1).

In the case of an ideal and incompressible fluid in deep water, the system mentioned can be written in the kind[2,3]

$$
\begin{gathered}
\rho \frac{d \mathbf{u}}{d t}=-\vec{\nabla}_{3} P-\rho \mathbf{g}+\left.\mathbf{f}(\mathbf{x}, t)\right|_{z=\eta(\mathbf{x}, t)}, \\
\vec{\nabla}_{3}(\mathbf{u})=0, \\
\left.u_{z}\right|_{z=\eta(\mathbf{x}, t)}=\frac{\partial \eta}{\partial t}+\left(\mathbf{u} \vec{\nabla}_{2} \eta\right), \\
\left.u_{z}\right|_{z=-\infty}=0 .
\end{gathered}
$$

where the following designations are used: $\rho(z, t)$ is the fluid density; $\mathbf{u}(\mathbf{x}, z, t)=\left(u_{x}, u_{y}, u_{z}\right)$ is the velocity field; $P(\mathbf{x}, z, t)$ is the atmospheric pressure; $g$ is the acceleration due to gravity; $f(\mathbf{x}, z, t)$ is the external forcing (surface tension, wind stress and so on); $\eta(\mathbf{x}, t)$ is the surface elevation field; $\mathbf{x}=(x, y)$ is the horizontal coordinates vector; $z$ is the vertical coordinate up-directed; $\vec{\nabla}_{2}=(\partial / \partial x, \partial / \partial y)$ is the horizontal gradient vector; $\vec{\nabla}_{3}=\left(\vec{\nabla}_{2}, \partial / \partial z\right)$ is the full gradient, and the full time-derivative operator is defined as $d(\ldots) / d t=\left(\partial / \partial t+\mathbf{u} \vec{\nabla}_{3}\right)(\ldots)$.

Due to nonlinearity of the problem, a numerical solution of system (1.2)-(1.5) is even of great difficulties, to say nothing about analytical solutions. Nevertheless, to present time evolution mechanisms $N L$ and $I N$ have certain and widely recognized spectral representations, $N L(S)$ and $I N(S)$, which are derived from equations (1.2)-(1.5) (for references, see[2-4]). However, such a kind representation $D I S(S)$ is absent for the dissipation mechanism. This fact has several important causes, the main of which are the following.

In the theoretical aspect, the general cause is provided by the fact that numerous physical processes resulting in the wave energy dissipation are related to different kinds of instabilities, including disruption of the air-sea interface surface (wave breaking, white capping, air-bubble intrusion, and others). All these processes form a specific multi-scale or, more exactly, cascade mechanism of wave dissipation. In this cascade, some "fast" events (mainly, wave breaking) play a role of primary process generating different kinds of small-scale motions. But the actual, "slow" dissipation of wave spectral components takes place on the background of these motions (as far as the spectrum is defined on a large time scale, only). In such a case, application of the standard equations, for example (1.2)-(1.5), requires their essential modification and a proper mathematical method of their solution, and this does cause the main theoretical problems (for example, see[1]. These problems result in the fact that the widely recognized and theoretically justified model for the wave energy dissipation mechanism does not exist.

Despite a presence of numerous theoretical papers, for example, statistical estimations in Longuet-Higgins[5], Battjes and Janssen[6], direct derivations in Hasselmann[1], dimensional considerations in Phillips[7], Polnikov[8], Zaslavskii[9], Donelan[10], and numerical simulations in Chalikov and Sheinin[11], Zakharov et al.[12], a sufficiently convincing solution of this point was not found. Unfortunately, this issue is rarely discussed in scientific literature from a general and physical point of view. This fact slows down the construction of justified formulation for a spectral representation of dissipation function $D I S(S)$. This paper is dedicated to accelerate solution of the point.

\section{General Ideas and Objectives of the Work}

The most important and even the key factor complicating the solution of the problem is the fact that any experimental investigations of the wave dissipation processes are principally restricted. This occurs due to the existence of numerous, poorly observed and therefore hardly measurable processes taking place on the background of visible dissipation wave processes (mainly, wave breaking). Non-controlled instability of shear and orbital currents (on the background of stochastic waves) and permanent generation of turbulence both in the upper water layer and in the air layer nearby the interface can be attributed to the class of poorly observed processes.

Besides, the wave dissipation occurs on the background of permanent energy exchange between wind and waves ( $I N$ mechanism) and immeasurable nonlinear energy transfer between waves ( $N L$-mechanism). Therefore, the previous statement about the strong restriction of possibilities for experimental studying the whole package of wind-wave dissipation mechanisms becomes much more justified and, in our opinion, quite convincing.

Additional difficulty for measurements execution arises when it needs getting information about the wave energy dissipation rate in a spectral representation, i.e. representation of dissipation in a form of its distribution through wave spectrum components $\operatorname{DIS}(S(\sigma, \theta))$ rather than in a form of integral loss over a whole wave spectrum.

One can find a confirmation of the previous statement in the analysis of new, the most advanced experimental data regarding to wind wave dissipation features, found for the last 5-10 years. These results are represented in a rather comprehensive form in papers of Australian scientists (Banner and Young[13], Banner and Tian[14], Babanin et al.[15], Young and Babanin[16], Babanin and Westhuysen [17]) and in the comprehensive overview by Babanin[18]. The results mentioned above are substantially supplemented by the findings of American and French colleagues (for example, see references in[10-21] and [25], respectively.

First of all, it is seen from this literature that the overwhelming majority of experimental works, regarding to the wave dissipation, are mainly restricted by the research of wave breaking processes. Secondly, even the most certain result in this field, reported in[16] and presented in Fig. 1, does not answer the question about the nature and intensity of the wind-wave dissipation process.

Indeed, the so called "difference" between the wave spectra measured "before" and "after" breaking, shown in Fig.1, provides evidence that the energy of wave component corresponding to the spectrum peak is not lost totally. It is most likely that it is randomly distributed among other wave 
components. We do not dwell on a detailed analysis of this data (which is described in Appendix A). We only note that the result shown here emphasizes the validity of the above statement about the restriction of the experimental possibilities in research of the nature of wave dissipation.
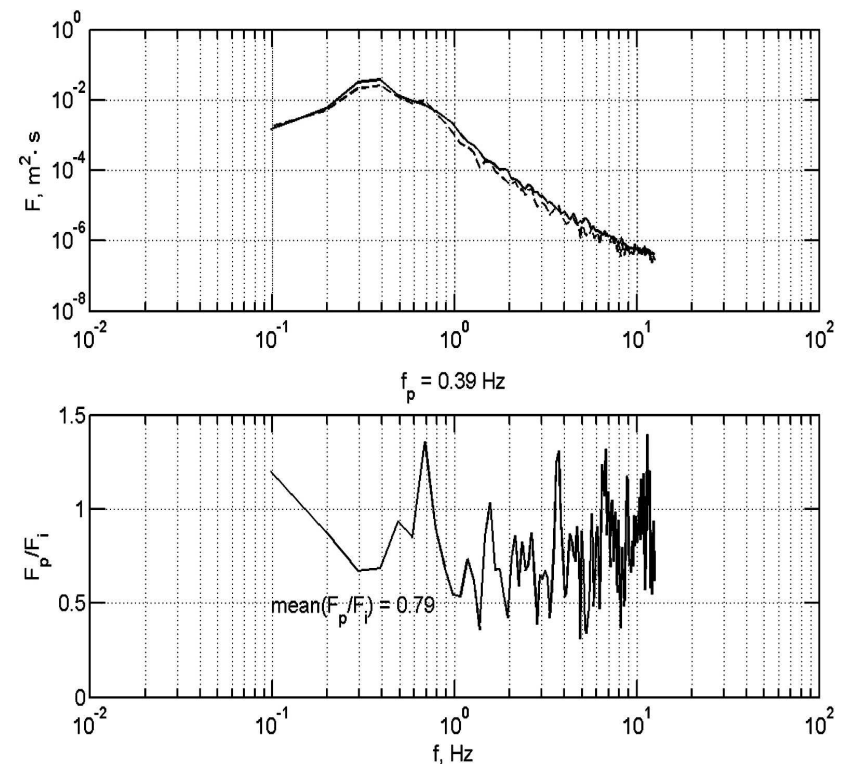

Figure 1. Top panel: Mean power spectrum of incipient-breaking (solid line) and post-breaking (dashed line) waves. Bottom panel: Ratio of the spectra shown in top panel (following to [16])

Nevertheless, despite of the difficulties enumerated, it this direction there is a remarkable series of theoretical and experimental results. Moreover, these results are for a long time used with one or another level of success in numerous wind waves numerical models of the form (1.1): WAM [22], WAVEWATCH (WW)[23], and others[24,25]. Herewith, the dissipation functions $D I S(S)$ used in these numerical models are poorly justified physically, in opposite to the relatively well developed and widely recognized parameterizations of $N L$ and $I N$ mechanisms.

In particular, the parameterizations of $D I S(S)$ in the form of quasi-linear spectral function

$$
\operatorname{DIS}(S)=\operatorname{int}(S) \cdot S,
$$

where $\operatorname{int}(S)$ is the some kind integral function of $S$, is widely used in such world-wide spread models as WAM, WW, and SWAN[17]. But it has a semi-phenomenological origin. The latter means that form (2.1) is constructed on the basis of general physical considerations which are not directly and unambiguously related to the measurements and fundamental physical equations. Therefore, the parameterizations $D I S(S)$ mentioned above are often and reasonably criticized from one or other viewpoints[2-4].

According to the aforesaid, the necessity of more logical and theoretically justified description of wind-wave energy dissipation processes in the spectral representation is still needed.

In addition, we note that for a long time there is an idea to construct the dissipation model basing on the statement that the surface-wave energy losses can occur due to the turbulent viscosity provided by the interaction of waves with the tur- bulence in the upper water layer[26]. It can be written in the form

$$
D I S(S)=v_{\mathrm{T}} k^{2} \mathrm{~S}
$$

where $v_{\mathrm{T}}(S)$ is the effective turbulent viscosity of the upper water layer, depending on the wave spectrum $S$. This idea was formally realized in several versions 25 years ago in[27] where some essential modelling preferences of the proposed representations of $D I S(S)$ were demonstrated. Later, this idea was developed by the author up to a semi-phenomenological theory. Unfortunately, the results were published partially [28], whilst the main derivations were retained in a manuscript of the author's doctoral dissertation[29]. Many years later, the author has sophisticated the final phenomenological result [4] and verified them successfully by means of their implementation in the mathematical shells of the world-wide known models mentioned above: WAM[30,31]. Nevertheless, the turbulent viscosity model is not widely recognized yet, as far as its full and regular justification does not exist. On the other hand, some experimental results in the field of studying the wave-induced turbulence have already appeared[20,32,33] and this fact stimulates our efforts in this field.

The issue of convincing justification of the spectral representation for wind-wave dissipation mechanism due to turbulence viscosity was many times discussed with A. Babanin, who is one of the leading investigators in this field. These discussions precisely stimulated the appearance of this paper. It is dedicated to gain the general understanding of the problem and definiteness in its solution. This is the main objective of the present work that has several constituents.

The first of the objectives is generalization of the point of view on the problem. With this in mind, the author performed a critical analysis of the most important approaches to the problem (theoretical works[5-12], and experimental researches[13-17]. This analysis showed that clear and physically well justified models of the wind-wave dissipation mechanism do not exist yet. In our mind, the failure of all previous attempts was provided by the wrong choice of the theoretical approaches used. For this reason, as an alternative to the present approaches, here we have tried to apply the full-scaled similarity method widely used in statistical hydromechanics.

The similarity approach, formulated in subsection $3 a$, allows us to construct the most general spectral form for phenomenological function $D I S(S)$ in the local representation in $S$, which, under some assumptions, is given as

$$
\operatorname{DIS}(S)=\text { fun } 1 \cdot S^{2} \text {. }
$$

The detailed specification of unknown function fun 1 is presented in subsection $3 b$. As far as this particular form was repeatedly used by the author earlier (for references, see[34], the corresponding numerical evidence is presented in subsection $3 c$, demonstrating the preferences of the proposed representation for $D I S(S)$ with respect to the versions of $D I S(S)$ used in WAM and WW models (see Fig. 2 and Tab. 1 in subsection 3c).

The second, and rather self-contained constituent of our objective is the construction of theoretical justification of the 
found phenomenological representation, $D I S(S) \propto S^{2}$ (section 4). The goal of this part of work is to find such physical mechanism that supports the mentioned dependence $D I S(S)$. The mathematical approach described in[1] and the idea of the turbulent viscosity, as the main mechanism of wave energy losses, were applied to solve the problem. Eventually, the physical model of wind wave energy losses corresponding to form $D I S(S) \propto S^{2}$ was constructed on the basis of equations system (1.2)-(1.5). It is our opinion that this part of the work gives a convincing proof that the turbulent viscosity can be considered as the most general dissipation mechanism for wind waves represented in the spectral form.

The final overview for application of the results obtained is given in conclusive section 5 .

\section{Construction of Function $D I S(\sigma, \theta, W, S)$ by Means of Similarity Method}

Keeping in mind a multi-scale and stochastic feature of processes taking place at the air-sea interface, we state that the most effective method for solving the task posed is the similarity method (or method of dimensions consideration) widely used in different divisions of statistical hydromechanics[35].

In our case, the essence of similarity consists in the fact that dissipation rate $D I S(\sigma, \theta, W, S)$ realizing in the spectral domain, $(\sigma, \theta)$, is provided by local wind $\mathbf{W}$ and local wave spectrum intensity $S(\sigma, \theta)$. To the benefit of this statement the fact testifies that, in the case of stationary and homogeneous wind, the spectrum "tale" $\left(S(\sigma, \theta)\right.$ at $\left.\sigma>2 \sigma_{p}\right)$ has the fixed shape named as equilibrium spectrum $S_{e q}(\sigma, \theta)[1,2]$. Moreover, it is widely recognized (see the same references) that formation of an equilibrium shape for a spectrum tale is stipulated by the balance between input and dissipation rates of the kind

$$
I N(\sigma, W, S \text { eq })-D I S(\sigma, W, S \text { eq }) \approx 0
$$

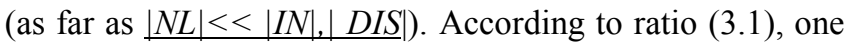
should expect a local feature of the dissipation function, by analogy to feature of the input one. The latter means that there are no integrated (cumulative) spectral terms in representation of function $D I S(S)$.

All these circumstances give basis for application of the dimensions consideration method for postulating an initial kind of function $\operatorname{DIS}(\sigma, \theta, W, S)$. Herewith, the physical nature of wave energy loss is quite unessential, as far as this approach has a phenomenological feature. The former will be needed at the stage of theoretical justification of the phenomenological result found by the similarity method. In this section, solution of the task posed is represented in the frame of full-scale similarity method for the first time.

\subsection{Phenomenological Construction of the General Kind of $D I S(\sigma, W, S)$}

The parameters of the task solved (and the system con- sidered) are as follows: the wave spectrum, $S(\sigma, \theta)$, the peak frequency of the spectrum, $\sigma_{p}$, the steepness of waves at the current frequency, $\varepsilon(\sigma)$, the phase speed of wave crests, which is of the order of $c_{p}=g / \sigma_{p}$, the local wind speed, $W$, and the gravity acceleration, $g$. Thus, in the task posed one can form a long series of dimensionless parameters:

- The dimensionless spectrum,

$$
\hat{S}(\sigma)=\sigma^{5} S(\sigma, \theta) / g^{2} \equiv \varepsilon^{2}(\sigma),
$$

- The dimensionless current frequency,

$$
\hat{\sigma}=\sigma / \sigma_{p},
$$

- The dimensionless phase speed of crests (or the wave age, $A$ )

$$
A=\hat{c}_{p}=c_{p} / W,
$$

- The dimensionless wind

$$
\hat{W}=W \sigma / g,
$$

and so on. To reach the aim posed, it needs to choose a combination of these parameters, providing for the general phenomenological representation of function $\operatorname{DIS}(\sigma, W, S)$.

Let us write presentation for $\operatorname{DIS}(\sigma, W, S)$ in the kind

$$
D I S(\sigma, W, S)=\text { const } \cdot \sigma S(\sigma, \theta) \cdot \Phi(\hat{S}, \hat{W}, A, \varepsilon, . .)
$$

where $\Phi(\hat{S}, \hat{W}, A, \varepsilon, .$.$) is the sought function of a whole ag-$ gregate of parameters mentioned above. With no account of the angular dependence of $D I S$, clarification of which is still not under consideration, expression (3.3) can be accepted as the most general representation of function $\operatorname{DIS}(\sigma, W, S)$. With the aim of finding the most simple form of function $\Phi(\ldots)$ among a multitude of its specifications, farther we will put one or another restraint to the representation of $\Phi(\ldots)$.

First of all, assuming that the dissipation function is the regular, growing, and local function of spectrum $S$, with no restriction of generality one can represent function $\Phi(\ldots)$ as a series to powers in spectrum of the following kind

$$
\Phi(\ldots)=\sum_{n} \alpha_{n}(A, \hat{\sigma}, \hat{W}, \varepsilon) \hat{S}^{n} .
$$

Now, take into account that a value of dimensionless spectrum $\hat{S}$ given by ratio (3.2a), in accordance with well recognized empirical data, is a small parameter of the system, having the order of Phillips' constant $\alpha_{p}$ [2], i.e.

$$
\hat{S} \cong \alpha_{p} \cong 0.01<<1 \text {. }
$$

In such a case, it immediately follows from (3.4) that representation for $\Phi(.$.$) can be restricted by several first terms$ of series (3.4), having a real physical sense.

Second, let us specify the meaning of really essential terms of series (3.4). To this end, it needs to attract the wellknown conception about equilibrium spectrum $S_{e q}(\sigma, \theta)$ realizing in the tail domain of the spectrum, defined by the ratio

$$
\hat{\sigma}>(2 \div 2.5) \text {. }
$$

The commonly recognized condition of existence of such a spectrum is the balance between input and dissipation mechanisms, given by equation (2.1). In turn, the commonly recognized representation for input function $I N(W, S)$ has the kind of linear in spectrum function $[2,3]$ 


$$
I N(W, S)=\beta(\sigma, \theta, \mathbf{W}) \sigma S(\sigma, \theta) .
$$

Coefficient $\beta(\ldots)$ in the right-hand side of (3.7) is the so called wave-growing increment depending on the parameters mentioned above. Specification of function $\beta(\ldots)$ is still not principal, as it is sufficient to accept that analytical representation of $\beta(\ldots)$ is fully defined[2,3].

In this case, it immediately follows from formula (1.1) for the source function that the mathematically similar, linear in spectrum $S$ summands of $I N(S)$ and $D I S(S)$ terms can be united to a single summand of SF. The final non-dimensional coefficient of united summand linear in $S$ can be treated as "the effective" increment $\beta(\ldots)$. (This can be done with no loss of physical sense of SF terms). It is just this increment which is commonly used in the input function $I N$. This circumstance allows us to exclude the linear in spectrum summand of series (3.4) for the representation of function $D I S(S)$. Consequently, the only term of series (3.4), which has certain physical sense, is the summand with the second power in spectrum $S$. As a result, with no loss for physical sense of SF, the general representation for dissipation function (3.4) is reduced to the form

$$
\begin{aligned}
D I S(\sigma, W, S) & =\sigma S(\sigma, \theta) \cdot \alpha_{1}(A, \hat{\sigma}, \hat{W}, \varepsilon) \hat{S} \\
& =\hat{\alpha}_{1}(A, \hat{\sigma}, \hat{W}, \varepsilon) \frac{\sigma^{6} S^{2}(\sigma, \theta)}{g^{2}}
\end{aligned}
$$

Representation (3.8) specifies fully the spectral dependence for function $D I S(S)$, and the rest ambiguity in $D I S$ is reduced to the new unknown function $\hat{\alpha}_{1}(\ldots)$ independent of $S$.

Thus, the problem of spectral representation of dissipation function is solved in the frame of phenomenological approach under assumption that this function is local in wave spectrum, i.e. there are no integral (cumulative) spectral terms. As it was shown above, in this representation there is not place neither for the linear in spectrum term (due to its mathematical similarity to the input term), nor for the terms having powers in spectrum higher than the second one (due to their negligibility).

\subsection{Specification of function $D I S(\sigma, \mathbf{W}, S)$}

Farther specification of the dissipation function is defined by the specification of the kind of auxiliary function $\alpha_{1}(A, \hat{\sigma}, \hat{W}, \varepsilon)$. It is realized by the following way.

First of all, let us write the condition of the equilibrium spectrum existence in the kind

$$
\left.[I N-D I S]\right|_{S=S_{e q}} \approx 0
$$

where it is implied that condition (3.9) is valid in domain (3.6), only. Substitution of (3.7) and (3.8) into (3.9) leads, in fact, to equation for spectrum shape $S_{e q}(\sigma, \theta)$, solution of which has the kind ${ }^{2}$

$$
S_{e q}(\sigma, \theta)=\frac{\beta(\sigma, \theta, \mathbf{W})}{\alpha_{1}(A, \hat{\sigma}, \hat{W}, \varepsilon)} g^{2} \sigma^{-5}
$$

According to (3.10), specification of $\alpha_{1}(A, \hat{\sigma}, \hat{W}, \varepsilon)$ is unequivocally defined by putting the form of equilibrium

\footnotetext{
${ }^{2}$ By the way, this is the key algebraic advantage of the quadratic form for $D I S(S)$.
}

spectrum $S_{e q}(\sigma, \theta)$. So, this form is a constructive element of the model under derivations, together with the kind of representation for wave growth increment $\beta(.$.$) .$

Combining the known models proposed by Phillips[36] and Toba[37], for the equilibrium spectrum shape one can get the following united kind

$$
S_{e q}(\sigma, \theta)=\operatorname{const}(n) \cdot g^{2} \sigma^{-5} \cdot \hat{W}^{n}
$$

allowing variability of power $n$ for the dimensionless wind in a wide range of magnitudes. Herewith, value $n=1$ corresponds to Toba's spectrum, whilst $n=0$ does to Phillips' one.

As a result, substitution of (3.11) into (3.10) gives the following solution

$$
\alpha_{1}(A, \hat{\sigma}, \hat{W}, \varepsilon)=C_{d i s}(n) \phi(A, \hat{\sigma}, \varepsilon) \beta_{M}(\sigma, \theta, \mathbf{W}) / \hat{W}^{n}
$$

where the fitting coefficient, $C_{d i s}(n)$, depending on the excepted form for $S_{e q}(\sigma, \theta)$, is shown explicitly, and the new dimensionless function, $\phi(A, \hat{\sigma}, \varepsilon)$, is extracted, which is not defined yet.

It should be mentioned here that due to a possible change the sign of increment $\beta(\sigma, \theta, \mathbf{W})$ (for swell components or low frequency components of spectrum, overtaking the local wind), the factor $\beta_{M}(\sigma, \theta, \mathbf{W})$ in (3.12) should be taken in the form of simple modification for increment $\beta(\sigma, \theta, \mathbf{W})$

$$
\beta_{M}(\sigma, \theta, \mathbf{W})=\max \left[\beta_{L}, \beta(\sigma, \theta, \mathbf{W})\right]
$$

Here, the standard designation $\max [a, b]$ means a choice of maximum among values under the brackets. This modification is needed to secure a proper sign for the dissipation function. The positive constant, $\beta_{L}$, is the so call "background" value of factor $\beta_{M}$, defined during the model fitting process (see subsection 3.4 below)

Expressions (3.12-3.13) finalize, in fact, the part of specification of dissipation function $\operatorname{DIS}(\ldots)$ regarding to its dependences both on spectrum $S$ and local wind $\mathbf{W}$. This fact ensures independence of function $\phi(A, \hat{\sigma}, \varepsilon)$ of $S$ and $\mathbf{W}$. Moreover, in the domain given by (3.5), it is quite acceptable the following asymptotic $\phi(A, \hat{\sigma}, \varepsilon) \approx 1$, as far as the proper representation for $D I S(. .$.$) describes fully the formation of$ known empirical tale of wave spectrum.

In the vicinity of the spectrum peak, i.e. in the domain given by the ratio $0.5<\hat{\sigma}<2$, the specification of $D I S(\ldots)$, resulting from the kind of function $\phi(A, \hat{\sigma}, \varepsilon)$, needs attraction of new empirical information and phenomenological hypothesis regarding to behaviour of function $D I S(\ldots)$ in this domain. It was proposed earlier $[28,4]$ in the following final form

$$
\phi(A, \hat{\sigma}, \varepsilon)=\phi\left(\theta, \theta_{w}, \hat{\sigma}\right)=\max \left[0,\left(1-c_{\sigma} \hat{\sigma}^{-1}\right] T\left(\theta, \theta_{w}, \hat{\sigma}\right)\right.
$$

with the angular function

$$
T\left(\theta, \theta_{w}, \hat{\sigma}\right)=\left\{1+4 \sin ^{2}\left(\frac{\theta-\theta_{w}}{2}\right) \hat{\sigma}\right\} \max \left[1,1-\cos \left(\theta-\theta_{w}\right)\right]
$$

where $c_{\sigma}$ is the additional parameter of the dissipation model, $\theta_{w}$ is the local wind direction, and the standard designation $\max [a, b]$ means the choice of maximum among values under the brackets. It should be especially mentioned here the two-mode angular representation (3.15), well cor- 
responding to the recent empirical results[16] regarding to angular distribution of DIS (for details, see Appendix A).

Thus, formulas (3.8) and (3.12)-(3.15) finalize totally the proposed phenomenological parameterization of the dissipation function. As it has turned later, this parameterization reflects (and does not contradict to) the most reliable of modern empirical data[16], excluding the effects of cumulative and threshold features of $D I S(S)$, discussed in details in Appendix A.

\subsection{Physical Meaning of the Parameters and Effectiveness of Phenomenological Function $\operatorname{DIS}(\sigma, \mathbf{W}, S)$}

Moving the theoretical justification of the proposed spectral representation, $D I S(S) \propto S^{2}$, to the after-following separate consideration (section 4 ), let us give here the short description of meaning of the parameters introduced above, and show some evidence of new parameterization preference with respect to the analogues used in WAM and WW.

First of all, note that in the dissipation parameterization proposed above there are only four free varying values: $C_{d i s}$, $\beta_{L}, c_{\sigma}$, and $n$.

The meaning of the fitting coefficient $C_{\text {dis }}$ is evident: it regulates the dissipation intensity. This parameter is inevitable in any model that has the SF represented in the additive form (1.1). Moreover, $C_{d i s}$ is the most actively varied in the course of fitting the model given by the wave spectrum evolution equation of the form (1.1).

Parameter $n$ is the next in importance. Its meaning is evident also: it is responsible for the shape of an "expected" equilibrium spectrum, which is provided by the numerical model. Value $n=1$ supports the Toba's equilibrium spectrum, $S_{e q}(\sigma) \propto W g \sigma^{-4}$, whilst value $n=0$ does the Phillips' spectrum, $S_{e q}(\sigma) \propto g^{2} \sigma^{-5}$. Consequently, by means of varying the value of $n$, users of the model are free to choose the expected shape of numerical equilibrium spectrum. Hereof, by the way, it follows that the falling law "-4" of the spectrum tale (i.e. $S(\sigma) \propto \sigma^{-4}$ ) is easily realized with no account of nonlinear term $N L$ (in opposite to the reasons discussed in[7], and[38]. So, the proposed approach gives an additional freedom for physical treating the Toba's spectrum formation.

Side by side with $n$, parameter $c_{\sigma}$ is the element of fine fitting the calculating spectrum shape. It allows varying the dissipation intensity in the spectral peak domain and, at some extent, the dissipation intensity in the spectrum-tale domain. In fact, existence of this coefficient corresponds to the empirical data[16] that dissipation intensity at the spectrum tale is defined by the intensity of the dominant waves breaking. For example, our simulations show $[30,31]$ that varying $c_{\sigma}$ allows, at some extent, to change the mean frequency, $\sigma_{m}$, which is one of the integrated characteristics of the spectrum, given by the ratio

$$
\sigma_{m}=\iint \sigma S(\sigma, \theta) d \sigma d \theta / \iint S(\sigma, \theta) d \sigma d \theta
$$

Relationship between $\sigma_{p}$ and $\sigma_{m}$ is important as one of the "checked" magnitudes analysed in the course of numerical model verification [34]. By the way, this relationship is defined by a choice of parameter $n$, as well.

Finally, several words about parameter $\beta_{L}$. In the light of the said above for explanation of formula (3.14), this parameter is used to regulate the dissipation intensity during the processes of rapid change of wind velocity vector $\mathbf{W}$ (turning or going down), corresponding to the transition of wind components to a swell. For such components, the value of increment $\beta(\sigma, \theta, \mathbf{W})$ undergoes the rapid decrease resulting in diminishing the intensity of wave breaking. Herewith, a certain extent of the background turbulence in the upper water layer is still retained what ensures a remarkable attenuation of the wind-wave components that became the swell. Together with the wave generation process, this attenuation permits waves to turn faster to the new wind direction (see detailed simulations in Polnikov 2005). These situations are poorly described by the models with the traditional dissipation functions (WAM and WW), where the background dissipation is simply absent. The choice of the order of value $\beta_{L}$ is defined by the empirical and numerical simulation results $[4,11]$.

In conclusion we note that phenomenological function of dissipation angular distribution $T\left(\sigma, \theta, \theta_{w}\right)$ of kind (3.15) (which is totally made "by hands" still in[28], corresponds rather well to the empirical data[16] described in Appendix A.

Dwell now on the preferences of new dissipation function, realized during numerical modelling of wind waves. First of all, we note that the only possibility to proof these preferences is the procedure of comparative verification. Such kind verification was performed in papers[30,31,34] where the models WAM and WW were accepted as the reference ones. The comparative verification procedure was performed for the integrated characteristics of wave field: the significant wave height $H_{\mathrm{S}}$, and the mean period $\mathrm{T}_{\mathrm{m}}$, only. They were calculated both with the original version of the models mentioned and with their modifications made by replacing the corresponding SF in the both models (for details, see references).

Note that in the both cases, modification of SF is related to the change of all three terms. Herewith, the change of terms $N L$ and $I N$ does not practically change the physics of SF, whilst the change of $D I S$, in opposite, does it radically. Variation of the models modification, realized by means of a consecutive and separate change of the SF-terms, permitted us to find that all principal differences in accuracy of wavefield calculations are caused by the change of the $D I S$-term [31].

In all the mentioned papers, it was shown for a great data base that the change of $D I S$-term resulting in increasing the simulation accuracy for significant wave height $H_{s}$ on $15-20 \%$ and for mean period $T_{m}$ up to $50 \%$ (see in[31].

For more convincingness, we plot here the time history $H_{s}(t)$ (Fig. 2) which illustrates that the change of SF (the model NEW) results in better description of the extreme wave-heights and their sequential attenuation. Such attenua- 
tion is evidently related to the change of the local wind at the point under consideration, and these situations are better described by our $D I S$-term.

Here we have no place (and necessity) for a full analysis of the comparison method and for demonstrating more details, which can be found in[30,31,34]. At the moment it is important to emphasize only the fact of remarkable increasing of simulations accuracy what is achieved simply by changing the physical content of the source functions used in WAM and WW. Herewith, the key item of this changing is the dissipation term, detailed study of which is the main aim of this work.

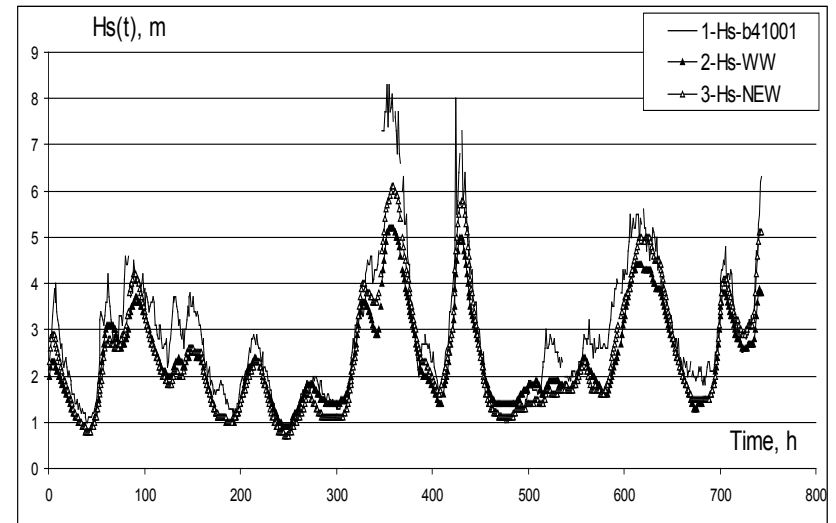

Figure 2. The time history of significant wave height $H_{\mathrm{s}}(t)$ at the location point of buoy 41001 in the North Atlantic for the period January 2006: 1buoy observations, 2 - results of modelling with the model WW, 3 - results of modelling with the modified model WW[31]

For completeness of the problem solution, it is left to consider the point of physical justification the quadratic dependence, $D I S(S) \propto S^{2}$, resulting in the mentioned positive results of verification.

\section{Theoretical Justification of Form $D I S(S) \propto S^{2}$. the Eddy Viscosity Model}

\subsection{Reynolds Stresses and the Main Fundamentals of the Model}

The main fundamental of the proposed model states that, on the scales of validity the evolution equation for wind waves in the spectral form, the central and most general cause of the wind-wave energy dissipation is the turbulence of the upper water layer. Herewith, the specification of the processes producing the turbulence is insignificant.

It is evident that a reasonable part of the turbulence intensity is provided by the wave breaking processes. Though, it is also clear that a multitude of accompanying processes (mentioned earlier in section 1) generates a cascade of chaotic motions with no determined scales, i.e. the turbulence motions. In our mind, these motions make the main contribution to the wind-wave dissipation. In other words, the proposed theoretical approach is based on the accounting all dissipative processes resulting in the turbulence production in the upper water layer.

According to the said, without any restriction of generality, the field of currents $\mathbf{u}(\mathbf{x}, t)$ can be written in a wavy water layer in the form of two constituents

$$
\mathbf{u}(\mathbf{x}, t)=\mathbf{u}_{w}(\mathbf{x}, t)+\mathbf{u}^{\prime}(\mathbf{x}, t) .
$$

The first summand in the right-hand side (farther, the r.h.s.) of (4.1), $\mathbf{u}_{w}$, we treat as the potential motion attributed to the wind waves, whilst the second summand, $\mathbf{u}^{\prime}$, is treated as the turbulent constituent of full currents field, totally uncorrelated with $\mathbf{u}_{w}$ in the statistical sense. Herewith, it is important to note that a corresponding representation for the elevation field $\eta(\mathbf{x}, t)$ in not necessary, if one accepts the Hasselmann's statement about "weakness in mean" for processes of the water surface ruptures[1].

Now, substituting representation (4.1) into initial equation (1.2) (with no external forces) and into (1.4), by means of averaging over turbulent scales we get the following system (subindex $w$ is omitted)

$$
\begin{gathered}
\frac{\partial \bar{u}_{i}}{\partial t}+\sum_{j} \bar{u}_{j} \frac{\partial \bar{u}_{i}}{\partial x_{j}}=-g \delta_{i, 3}-\sum_{j} \frac{\partial<u_{i}^{\prime} u_{j}^{\prime}>}{\partial x_{j}} \\
\frac{\partial \bar{\eta}}{\partial t}+\sum_{i=1,2} \bar{u}_{i} \frac{\partial \bar{\eta}}{\partial x_{i}}=\bar{u}_{3} .
\end{gathered}
$$

Here, the tensor form of equations is used; the mean wave variables are denoted by the bar in above (farther, the bar will be omitted for simplicity); and brackets $<\ldots>$ are the symbol of averaging over the turbulent scales. Due to nonlinearity of the system, a new term is appearing in the r.h.s. of (4.2):

$$
P_{i} \equiv \sum_{j} \frac{\partial<u_{i}^{\prime} u_{j}^{\prime}>}{\partial x_{j}} \text {. }
$$

Physical meaning of the term $\mathbf{P}$ is the forcing which results in the wave motion dissipation [1].

Numerator $\left\langle u_{i}^{\prime} u_{j}^{\prime}\right\rangle \equiv \tau_{i j}$ in expression (4.4) is the well-known magnitude called the Reynolds stress tensor[35] written here in the normalization to the water density. Methods of parameterization for $\tau_{i j}$ are also rather well developed. Therefore, to construct the theory, it needs to specify $\tau_{i j}$ in terms of the large-scale, i.e. wave motion variables, $\eta$ and $\mathbf{u}$, and to ascribe to this specification a certain physical content. After this, with the aim of getting the final result, one can use the mathematics of[1].

To reach the aim posed, let us accept a series of additional fundamentals. Firstly, following to[1], we accept the fundamental about "weakness of distortions in mean". It allows us to retain the common meaning for the wave profile, $\eta(\mathbf{x}, t)$, and potential wave motion, $\mathbf{u}(\mathbf{x}, t)$, and to introduce any derivatives for these variables. Secondly, we suppose that the magnitude of nonlinear stresses $\tau_{i j}$ does essentially exceed the weak dynamic nonlinearity of the system, described by the second terms in the 1.h.s. of (4.2) and (4.3). Hereby, we postulate the fundamental of the "strong" turbulence. Thus, system (4.2), (4.3) takes the following kind

$$
\begin{gathered}
\frac{\partial u_{i}}{\partial t}+g \delta_{i, 3}=-P_{i}(\mathbf{u}, \eta), \\
\frac{\partial \eta}{\partial t}=u_{3} .
\end{gathered}
$$




\subsection{Phenomenological Closure of Reynolds Stress}

Now, we formulate the main grounds of our concept for the procedure of the Reynolds stress closure, the aim of which is to express the turbulent characteristic $\tau_{i j}$ via wave variables $\eta$ and $\mathbf{u}$.

To do this, we remind the version of such closure, consisting in using the well-known Prandtl's conception of random mixing length $\lambda^{\prime}$ [35]. In the simplest case of near-wall turbulence, the Prandtl's approach results in the Reynolds stress closure in the form of quadratic function in the velocity field

$$
\begin{aligned}
\tau_{i j} & =<\lambda_{i}^{\prime}\left(\partial u_{i} / \partial x_{3}\right) \lambda_{j}^{\prime}\left(\partial u_{j} / \partial x_{3}\right)> \\
& =<\lambda_{i}^{\prime} \lambda_{j}^{\prime}>\left(\partial u_{i} / \partial x_{3}\right)\left(\partial u_{j} / \partial x_{3}\right)
\end{aligned}
$$

In our case, besides of the velocity field instability, the turbulence is provided by the variability of the interface surface. Therefore, representation (4.7) should be properly generalized. As a certain result of this generalization, the distortion forcing $-P_{i}(\mathbf{u}, \eta)$ can be represented, for example, by the following quadratic function of wave variables $\mathbf{u}$ and $\eta$

$$
\begin{aligned}
& -P_{i}(\mathbf{u}, \eta)=<\sum_{j} \frac{\partial}{\partial x_{j}}\left\{\left[\lambda_{i}^{\prime}\left(\partial u_{i} / \partial x_{3}\right)+v_{i}^{\prime}\left(\partial \eta / \partial x_{i}\right)\right] \times\right. \\
& \left.\times\left[\lambda_{j}^{\prime}\left(\partial u_{j} / \partial x_{3}\right)+v_{j}^{\prime}\left(\partial \eta / \partial x_{i}\right)\right]\right\}>
\end{aligned}
$$

where the random values $v_{i}^{\prime}$ have the meaning of mixing velocities.

Here it is important to note that we do not know real processes of the turbulence formation in the upper water layer. Therefore, there is no sense to construct any more complicated and specified approximation for distortion $\mathbf{P}$ via physical variables $\mathbf{u}$ and $\eta$, as it was done in the previous papers by the author[28,29]. It is essential only that representation (4.8) retains the following main features of the problem: nonlinearity of the distortion forcing $\mathbf{P}$ in the wave variables, and dependence $\mathbf{P}$ on the gradients on both velocity field $\mathbf{u}(\mathbf{x}, z, t)$ and elevation field $\eta(\mathbf{x}, t)$. As it will be shown below, the nonlinear feature of the distortion forcing gives sufficient grounds for finding the general kind of the spectral representation for $D I S(S)$.

\subsection{General Kind of the Problem Solution in the Spectral Representation}

In the case of deep water, the full system of equations to be solved includes dynamic equations (4.5), (4.6), written at the interface surface $\eta(\mathbf{x}, t)$, and equations (1.4), (1.5) valid in a whole volume of fluid. The proper system of equations, written in the potential motion approximation, has the kind

$$
\begin{gathered}
\dot{\Phi}+g \eta=-\hat{P}(\eta, \Phi), \\
\dot{\eta}=\frac{\partial \Phi}{\partial z}, \\
\sum_{i} \frac{\partial^{2} \varphi}{\partial x_{i}^{2}}=0 \text { and }\left.\frac{\partial \varphi}{\partial z}\right|_{z=-\infty}=0 .
\end{gathered}
$$

Here, the distortion function $\hat{P}(\eta, \Phi)=\left(\vec{\nabla}_{3}\right)^{-1}[P(\eta, \mathbf{u})]$ represents the certain modification of function (4.8), induced by the procedure of transition to the scalar potential of velocity field, $\varphi(\mathbf{x}, z, t)$ whilst $\Phi \equiv \varphi(\mathbf{x}, \eta(\mathbf{x}), t)$ is the potential at the elevation surface.

Farther, the variables are to be written in the form of the Fourier-Stiltjes decomposition in the wave vector space (see details in[1,38])

$$
\begin{gathered}
\eta(\mathbf{x}, t)=\text { const } \cdot \int_{\mathbf{k}} \exp [i(\mathbf{k x})] \eta_{\mathbf{k}}(t) d \mathbf{k} ; \\
\varphi(\mathbf{x}, z, t)=\text { const } \cdot \int_{\mathbf{k}} \exp [i(\mathbf{k x})] f(z) \varphi_{\mathbf{k}}(t) d \mathbf{k}
\end{gathered}
$$

where $\mathbf{k}$ is the wave vector, and $f(z)$ is the vertical structure function of the potential, to be found from two equations (4.11). In our case, $f(z)=\exp (-k z)$. After substitution of representations (4.12) into (4.9)-(4.11), equations (4.11) result in the mentioned structure function $f(z)$, and two other equations get the kind

$$
\begin{aligned}
\dot{\Phi}_{\mathbf{k}}+g \eta_{\mathbf{k}} & =-P_{\mathbf{k}}\left(\eta_{\mathbf{k}}, \Phi_{\mathbf{k}}\right), \\
\dot{\eta}_{\mathbf{k}} & =k \Phi_{\mathbf{k}} .
\end{aligned}
$$

Here, $P_{\mathbf{k}}\left(\eta_{\mathbf{k}}, \Phi_{\mathbf{k}}\right) \equiv F^{-1}[\hat{P}(\eta, \Phi)]$, and the operator $F^{-1}$ means the inverse Fourier transform applied to function $\hat{P}(\eta, \Phi)$. The latter procedure is commonly used in the course of transition to the final equations written for the Fourier components, $\eta_{k}$ and $\Phi_{k}$ (for details, see $[38,39]$ ).

Here we should especially note that the inverse Fourier transform procedure needs a calculation of integrals of the kind

$$
\int_{\mathbf{k}} d \mathbf{x}\left[\exp (-i \mathbf{k} \mathbf{x})<\lambda_{i}^{\prime} \lambda_{j}^{\prime} \int_{\mathbf{k}_{1} \mathbf{k}_{2}} \phi\left(\mathbf{k}_{1}, \mathbf{k}_{2}\right) \exp \left(i \mathbf{k}_{1} \mathbf{x}\right) \exp \left(i \mathbf{k}_{2} \mathbf{x}\right) \eta_{\mathbf{k}_{1}} \eta_{\mathbf{k}_{2}} d \mathbf{k}_{1} d \mathbf{k}_{2}>\right]
$$

which are typical in the nonlinear theory for waves $[38,39]$. In the traditional theory, the form (4.15) gets the final expressions with the three-wave resonances. These resonances are defined by the factor $\delta\left(\mathbf{k}_{1} \pm \mathbf{k}_{2} \pm \mathbf{k}\right)$ under the sign of the final integral, which is provided by the analytical integration in (4.15) on $d \mathbf{x}$. But in our case, such resonances can result in the cumulative terms in the dissipation function, which are not desirable (see discussion in section 5).

To avoid appearing the cumulative terms in the final result, we use the principal difference between our expression (4.15) and the analogous one appearing in the traditional (conservative) nonlinear theory. In our case, this difference consists in the fact that the turbulent-scale averaging operator, given by the brackets $\langle\ldots\rangle$, takes place under the integral in (4.15). This fact allows us to accept the following hypothesis: the random multipliers of the closure (4.8), standing under the averaging brackets in (4.15) (alike $\lambda_{i}^{\prime} \lambda_{j}^{\prime}$ and analogous ones), due to their random small-scale feature, provide a radical rearrangement of the phase (exponential) multipliers in the integrand of (4.15), resulting in the non-resonance feature of interaction between wave and turbulence. This hypothesis simply means a postulating the following rule for statistical averaging in the integrals of kind (4.15):

$$
\begin{aligned}
& \int_{\mathbf{k}} d \mathbf{x}\left[\exp (-i \mathbf{k} \mathbf{x})<\lambda_{1}^{\prime} \lambda_{j} \iint_{\mathbf{k}_{1}} \phi\left(\mathbf{k}_{2}, \mathbf{k}_{1}\right) \exp \left(i \mathbf{k}_{1} \mathbf{x}\right) \exp \left(i \mathbf{k}_{2} \mathbf{x}\right) \eta_{\mathbf{k}_{1}} \eta_{\mathbf{k}_{2}} d \mathbf{k}_{1} d \mathbf{k}_{2}>\right]= \\
& =\int_{\mathbf{k}_{1}} \int_{\mathbf{k}_{2}} L\left(\mathbf{k}, \mathbf{k}_{1}, \mathbf{k}_{2}\right) \eta_{\mathbf{k}_{1}} \eta_{\mathbf{k}_{2}} d \mathbf{k}_{1} d \mathbf{k}_{2}
\end{aligned}
$$

where $L\left(\mathbf{k}, \mathbf{k}_{1}, \mathbf{k}_{2}\right)$ is the regular function of its arguments. 
Acceptance of this hypothesis allows avoiding the resonance (cumulative) forms in the final expression for nonlinear terms (4.15) and consider distortion forcing $P_{\mathbf{k}}\left(\eta_{\mathbf{k}}, \Phi_{\mathbf{k}}\right)$ in equation (4.13) as a regular quadratic form of Fourier variables $\eta_{\mathbf{k}}$ and $\dot{\eta}_{\mathbf{k}}$, alike in (4.16). In such case, the system (4.13), (4.14) is reduced to the standard equation for an oscillator with a weak and regular disturbance (see analogues in $[1,38]$ )

$$
\ddot{\eta}_{\mathrm{k}}+g k \eta_{\mathrm{k}}=-k P_{\mathrm{k}}\left(\eta_{\mathrm{k}}, \dot{\eta}_{\mathrm{k}}\right)
$$

The solution of equation (4.17) can be found in the spectral form by means of the method described in (Hasselmann 1974), which is very close to the standard method of the weak turbulence, presented for the first time in[38] and in more details in[39].

Moving the explanation algebra to Appendix B, here we only note that the quadratic form of distortion function $P_{\mathbf{k}}\left(\eta_{\mathbf{k}}, \dot{\eta}_{\mathbf{k}}\right)$ leads to the solution of (4.17) of the kind

$$
D I S(S, \mathbf{k}, \sigma)=\sum_{n=2}^{N} \alpha_{n}(\mathbf{k}, \sigma) F_{n}[S(\mathbf{k})],
$$

where $F_{n}[S(\mathbf{k})]$ is the functional to $n$-th power in the wave spectrum $S(\mathbf{k})$. As seen, expression (4.18) is fully equivalent to the functional representation of $D I S(S)$ in initial formulas (3.3)-(3.4) of the phenomenological similarity method. This equivalence of the spectral representations for dissipation function $\operatorname{DIS}(S)$ in both approaches finalizes the sought theoretical justification of the similarity method used (for details, see Appendix B).

In conclusion of this section, we should especially note that the main fundamental of the eddy viscosity model, resulting in (4.18), is nothing else the nonlinear closure for Reynolds stress of kind (4.8) (or any other quadratic form), accepted above. Consequently, both result (4.18) and the results of section 3 have the proper physical justification. These results make a basis of the proposed model of the wave-energy losses due to the eddy viscosity of the upper water layer.

\section{Conclusions}

In conclusion of the paper, we resume shortly the main fundamentals of the proposed model for wind-wave dissipation, the range its applicability, and prospective for its further elaboration.

Firstly, about fundamentals. An explicit functional form for the dissipation term of SF can be found in the frame of the phenomenological similarity method (section 3). But the physical justification of the initial formulas of the similarity method, (3.2)-(3.4), heeds attraction of the basic hydrodynamic equations. This aim is reached in the frame of the proposed eddy viscosity model.

The main fundamental of the model consists in the assumption that on the scales of wave-spectrum evolution description, i.e. hundreds of the dominant periods, the main physical cause of the wave-spectrum component dissipation is the turbulence of the upper water layer, induced by the whole package of dissipative processes taking place at the air-sea interface. It is natural that laws of the turbulence formation are hardly known for us. Therefore, in the course of the model construction, the following two important assumptions are postulated: a) the nonlinear feature of the forcing resulting in the wave dissipation; and b) the specific modification of the phase structure for those Fourier- components of wave elevation and velocity, which are involved in the Reynolds stress closure. Acceptance of these presumptions is stipulated by the aim of obtaining observable final formulas. The point of their practical applicability is to be justified a posteriori by means of the verification procedure. Successful results of such procedure are presented in section 3 (Fig. 2) what, in fact, justifies all the assumptions accepted.

Secondly, about the scales of applicability of the approach proposed and treating the role of breaking events. This issue can be considered on the basis of the fact that functions $I N(S)$, $D I S(S)$, and $N L(S)$ are linear, quadratic, and cubic in the spectrum, respectively ${ }^{3}$. It is known that each power of spectrum in the SF-terms of equation (1.1) increases the temporal scales for the proper mechanism in $\varepsilon^{-2}$ times. Therefore, the input mechanism is the most fast one. This mechanism is balanced by the more slow dissipation mechanism what result in the equilibrium spectrum-tail formation in the high frequencies domain. But in the peak domain where $I N(S)$ and $D I S(S)$ have the cutting factors, the most slow and energy-conservative nonlinear mechanism, $N L(S)$, plays the most principal role (for details, see $[4,40]$ ). Thus, the issue of treating the role of very fast and strongly nonlinear breaking processes finds its solution based on the mentioned spectral consideration.

Really, the only logically justified matching the fast wave breaking processes with the rather slow wave-energy dissipation rate consists in the conclusion that the breaking means mainly the chaotic redistribution of the wave energy through the wave frequency band but do not the fact of wave-energy dissipation. Figure 1, being the direct measurement result of the wave-energy losses due to breaking, is the fine illustration to the said (see also text in section 1 and detailed comments in Appendix A). In this aspect, the known Hasselmann's hypothesis about "weakness of breaking in mean"[1] means, in fact, that the fast, non-conservative and nonlinear distortion during breaking is not directly related to a slow process of wave-energy losses, realized in the spectral representation. One may fix the wave energy losses only on the scales of the spectrum existence, i.e. on the scale of hundreds of dominant periods.

Herewith, it becomes clear that in the spectral representation for the wave dissipation mechanism, the threshold feature of the dissipation function, which is often fixed in the field and numerical experiments related to the study of separate breaking events $[12,16]$, should be totally smoothed due to statistical distribution of these event in time and space.

The said defines an applicability range for the dissipation

\footnotetext{
3 More details about roles of SF-terms and proper references can be found in $[4,40]$
} 
model discussed here: it does not describe fast (but rare at the fixed point of observation) separate breaking events; rather it is intended to description of the wave spectrum evolution realized on the scales of spatial-temporal variability of the whole statistical ensemble for a random wave field.

The last point is concerning a prospective of farther elaborating the theoretical justification of the model. Such a prospective is seen in the direction of specification the hypothesis about a phase-structure modification for the Fourier-components involved into the Reynolds stresses closure procedure (formula (4.16) in subsection 4.c). It is quite feasible that, in truth, some kind of the phase structure is conserved for the wave variables being under the turbulentscales averaging brackets in the integral of kind (4.15). Then, in the course of derivation the final expression for $D I S(S)$ of kind (4.18), functional $F_{n}[S(\mathbf{k})]$ could have the integral expressions of the convolution type. This change of functional kind for $D I S(S)$ is equivalent to appearing the cumulative dissipation term analogous to one proposed in[16] (see Appendix A). But in such a case, according to ratios (3.1) and (3.9), the functional form for input function $I N(S)$ should be properly modified, to save the widely accepted phenomenon of the equilibrium spectrum existence. Apparently, investigation of this point is a matter of a far future what is stipulated by an extraordinary complexity of verification the SF-terms of the cumulative type.

\section{ACKNOWLEDGEMENTS}

The author is grateful to Babanin A.V. for numerous discussions stimulating this paper, and to my colleagues co-working in the Russian Federal Program "World Ocean" for discussions. I acknowledge an interest of academicians of RAS Golitzin G.S. and Dymnikov V.P. to the problem. The work was supported in part by the Russian Federal Program "World Ocean" (State contract \#6) and by the Russian Foundation for Basic Research, \# 08-05-14524-ophi-c.

\section{Appendix A}

\section{Comments to results of [16]}

For completeness of the picture, describing the most important experimental results dealing with the study of breaking processes in waves, here we give some extractions from paper (Young and Babanin 2006) and our comments to their results.

Basing on the result of data processing, represented in Fig. 1 , and attracting other experimental facts, the motioned authors have recommended the following parameterization of $D I S(S)$, provided by the breaking of the dominant wave only $^{4}$

$$
\operatorname{DIS}(S)=a \sigma H\left[S(\sigma)-S_{t h}(\sigma)\right] A n(\sigma)+
$$

\footnotetext{
${ }^{4}$ Formula (A.1) is given with minor editing changes which are not principle in the physical sense.
}

$$
b \int_{\sigma_{p}}^{\sigma}\left(H\left[S(q)-S_{t h}(q)\right] A n(q)\right) d q .
$$

Here, $a$ and $b$ are the fitting constants; $H[\ldots]$ is the step-function given by the ratio

$$
H[x]=\left\{\begin{array}{l}
x \text { if } x>0 \\
0 \text { if } x \leq 0
\end{array} ;\right.
$$

$S_{t h}(\sigma)$ is the threshold spectrum level chosen especially; $A n(\sigma)$ is the dimensionless function of frequency, depending on the angular spreading of the wave spectrum. The integrated summand in the r.h.s. of (A.1) has the meaning of the "cumulative" impact of the low-frequency breaking on intensity of the dissipation for higher frequency components.

Results presented above can be characterized by the following way.

1) Dissipation function has the threshold feature;

2) Empirical function $D I S(S)$ is linear in spectrum;

3) The rate of losses in the frequency domain above the peak frequency $\sigma_{\mathrm{p}}$ has the cumulative feature, i.e. it is growing with the growth of losses at lower frequencies ${ }^{5}$.

Among the properties enumerated above, the linear dependence $\operatorname{DIS}(S)$ is the most essential empirical fact related to our aim. The threshold and cumulative features of empirical function $D I S(S)$ are interesting also, and they will be discussed later.

In a whole, it needs to acknowledge the importance and usefulness of the found empirical behaviour of function $D I S(S)$, provided by the breaking only. Herewith, in the frame of our statement about incompleteness of describing the whole package of the wave dissipation processes by the breaking only, the detailed assessment of the results represented is the following.

Firstly, the result shown in Fig. 1 does hardly allow getting the reliable and quantitative dependences $D I S(S)$, for the reason of inevitable statistical noise. Here we have two remarks.

Thus, even accepting, as the basis of analysis, the upper plot in Fig. 1, it evidently follows from the lower plot that the energy of breaking wave-components located at the spectral peak is not fully lost, it is rather chaotically spread among the others spectrum components.

Moreover, insufficient justification of the breaking-events separation in the wave-frequency band (see discussion of this point in [13-17]) leads automatically to a strong noise in the empirical information of the dissipation function dependence on frequency, $\operatorname{DIS}(\sigma, W, S)$. Therefore, it is not following by no way from Fig. 1 the conclusion about the cumulative feature of the dissipation processes, and the form given by the second term in (A.1) is not evident.

Secondly. From the point of view of the statistical ensemble meaning, which is very important for the spectrum

\footnotetext{
${ }^{5}$ It is worthwhile to mention here one more empirical fact which does not influence on the dependence $D I S(S)$ but relates to the angular dependence of dissipation intensity. This is the fact of the two-lobe profile for angular function $\operatorname{DIS}\left(\sigma, \theta, \theta_{w}\right)$ : the dissipation intensity is growing with initial growth of difference between direction of wave propagation $\theta$ and wind direction $\theta_{w}$, followed by the intensity going down with the farther growth of $\left(\theta-\theta_{w}\right)$
} 
definition, it seems that one may not separate the unique wave ensemble in two ensembles: "before breaking" and "after breaking". Such separation is the methodical incorrectness.

Really, per se of the wave-field ensemble definition, in any realization of the stochastic field (at any certain time moment), there is always a certain part of the field-square where the breaking takes place. Therefore, one may not separate "by hand" the realization with the breaking from the realization without the breaking, despite of the fact that there is no breaking at the point of measurement at the moment. Thereby the issue of the threshold feature of DIS(S) is closed. There is the threshold feature for a separate breaking, but the threshold feature of the breaking process is absent in the wave ensemble (due to a statistical structure of the field ensemble).

Thirdly. The breaking events are always too fast, to get reliable estimations of dynamics for the spectra "before breaking" and "after breaking". Such estimations can be realized for the frequencies far exceeding peak frequency $\sigma_{\mathrm{p}}$. But these frequencies cannot be separated from wave elevation records having their duration smaller several dominant periods: for this rather long time, the amplitudes of high frequency components "forget" about very fast breaking event (if any). It is true due to the stochastic feature of the wave field itself.

Fourthly. For each, very short period of the wave breaking at some space point, there are much more long periods of previous and past state of the wave field without breaking. Here the Hasselmann's postulate about "weakness of breaking impact"[1] on geometrical features of the interface surface becomes evident, as far as the wavy surface has the stochastic feature. By the way, just this postulate allows using the spectrum description for the stochastic elevations of the water surface.

The last two items say about the same: there is incomparability of the temporal scales for events of breaking (taking a small part of dominant period $T_{\mathrm{p}}=2 \pi / \sigma_{\mathrm{p}}$ ) and the scales of applicability of the spectral representation (hundreds of the dominant periods).

Fifth, let us say several words about representation (A.1), as it is. Omitting the threshold feature discussed earlier, we note that formula (A.1) has no explicit factor ensuring "autonomic" dependence of function DIS on local wind $W$. In principal, such factor could be included into coefficients $a$ and $b$. Herewith, due to the similarity consideration, the wind value could appear in $D I S$ via the proper dimensionless parameters of the system. It means that values of $a$ and $b$ should depend on the spectrum parameters. The absence of such dependence in (A.1) leads to the loss of information with respect to one, already known from the representations for function $\operatorname{DIS}(S, W)$ already used (in WAM or WW).

Herewith, the new information is given by the cumulative summand in (A.1). Though, due to importance of condition (3.9) for treating the equilibrium spectrum formation, existence of the cumulative summand in $D I S(S)$ demands a reconsideration of the spectral representation for input term
$I N(S)$ which has not such summand (yet). This inconsistence of the representations for $D I S(S)$ and $I N(S)$ is hardly acceptable, as far as it essentially complicates the treatment of the phenomenon of equilibrium spectral shape existence, the fact of which is empirically proved and widely recognized (see references in [2-3]).

Thus, interesting experimental representation (A.1), in our mind, does not practically result in any advancement in the problem of construction a realistic parameterization of function $D I S(\sigma, W, S)$ (but makes the latter even more difficult to treat). For this reason, it is not surprising very modest result of implementing formula (A.1) in testing experiments[42].

Hereby, present analysis of the results obtained on the basis of wave-breaking processes investigations[16] supports our earlier statement (section 2) about impossibility to study the whole package of real dissipative processes in waves by means of up-to-date experimental methods.

\section{Appendix B}

\section{Specification of equation (4.17) solution}

A short version of equation (4.17) solution in the spectral representation is the following. Following to[1], let us introduce the so called generalized variables

$$
a_{\mathbf{k}}^{s}=0.5\left(\eta_{\mathbf{k}}+s \frac{i}{\sigma(k)} \dot{\eta}_{\mathbf{k}}\right)
$$

(where $s= \pm$ and $\sigma(k)=(g k)^{1 / 2}$ ). Then, equation (4.17) can be rewritten in the well-known form with the time-derivative of the first order $[1,38]$ :

$$
\dot{a}_{\mathbf{k}}^{s}+i s \sigma(k) a_{\mathbf{k}}^{s}=-i s \sigma(k) P_{\mathbf{k}}\left(\eta_{\mathbf{k}}, \dot{\eta}_{\mathbf{k}}\right) / 2 g .
$$

Now, let us multiply equation (B.2) by the complex conjugated component $a_{\mathrm{k}}^{-s}$, make the sum of the resulting equation with the initial one, and make averaging the equation obtained over the wave ensemble. If we use the following definition of the wave spectrum from Hasselmann (1974)

$$
2<<a_{\mathbf{k}}^{s} a_{\mathbf{k}}^{s^{\prime}}>>=S(\mathbf{k}) \delta\left(s+s^{\prime}\right)
$$

where the double brackets $<<\ldots>>$ means the statistical averaging over wave ensemble, and $S(\mathbf{k})$ is the wave spectrum, we can get the following evolution equation for a wave spectrum

$$
\dot{S}(\mathbf{k}, t)=\frac{2 \sigma_{k}}{g} \operatorname{Im}<<P_{\mathbf{k}}\left(\eta_{\mathbf{k}}, \dot{\eta}_{\mathbf{k}}\right) a_{\mathbf{k}}^{-}>>.
$$

Now, making a certain specification of function $P_{\mathrm{k}}\left(\eta_{\mathrm{k}}, \dot{\eta}_{\mathrm{k}}\right)$ on the basis of closure (4.8), one can get the general kind of dissipation function $D I S(S)$ (4.18).

For the reason of qualitative representation of closure (4.8), there is no necessity to reproduce exactly all algebraic computations. Nevertheless, it is important to enumerate here for more convincingness the following circumstances enabling to get the final result:

a) The structure of the generalized variables $a_{\mathbf{k}}^{s}$ in (B.1) has the form of sum for the Fourier-components $\eta_{\mathbf{k}}$ and $\dot{\eta}_{\mathbf{k}}$. Initial form of distortion (4.8) is the products of the same 
sums what allows us to express the distortion via the generalized variables ;

b) Hypothesis (4.16) of the non-resonant feature for the wave-turbulence interactions allows the distortion forcing to be represented by a regular function of variables $a_{\mathbf{k}}^{s}$;

c) Hypothesis (4.16) can be used for any transformations of the integrands in the r.h.s. of equations (B.2) and (B.4) in the course of solution getting.

Items b) and c) allow to perform all actions with nonlinear summands of distortion function $P_{\mathbf{k}}\left(\eta_{\mathbf{k}}, \dot{\eta}_{\mathbf{k}}\right)$ without appearance any integrated convolution terms, in contrast to the resonant integrated terms typical in the conservative nonlinear theory (numerous details of such kind mathematics one can find in [38-41].

On the basis of items a)-c), for the aim of getting the general kind of solution, it is acceptable to write the final expression for $P_{\mathbf{k}}\left(\eta_{\mathbf{k}}, \Phi_{\mathbf{k}}\right)$ in the following simple form

$$
P_{\mathbf{k}}\left(\eta_{\mathbf{k}}, \Phi_{\mathbf{k}}\right)=\sum_{\mathbf{k}_{1}, \mathbf{k}_{2}} T\left(\mathbf{k}, \mathbf{k}_{1}, \mathbf{k}_{2}\right)\left[a_{\mathbf{k} 1}^{+} a_{\mathbf{k} 2}^{-}+a_{\mathbf{k} 1}^{-} a_{\mathbf{k} 2}^{+}\right] .
$$

Herewith, both an explicit form of factor $T\left(\mathbf{k}, \mathbf{k}_{1}, \mathbf{k}_{2}\right)$ and certain specification of quadratic function in the r.h.s. of (B.5) are not significant. From the mathematical point of view, the main feature of expression (B.5) is its nonlinear representation in amplitude $a_{\mathbf{k}}^{s}$.

Accepting (B.5), one can easily get the general kind of the r.h.s. of evolution equation (B.4) in the spectral form. Really, substitution of (B.5) into the r.h.s. of (B.4) results in the sum of the third statistical moments in amplitudes $a_{\mathbf{k}}^{s}$ of the kind $\left.<<a_{\mathbf{k} 1}^{s 1} a_{\mathbf{k} 2}^{s 2} a_{\mathbf{k} 3}^{s 3}\right\rangle>$. No of these moments can be expressed directly via spectral function $S(\mathbf{k})$ which is the even function to powers of the wave amplitudes. In such case, it needs to write and solve a proper equation for each the third moments by means of using basic equation (B.2) (for details, see [38-41]).

The third moment will be expressed via a set of the fourth moments of the kind $<<a_{\mathbf{k} 1}^{s 1} a_{\mathbf{k} 2}^{s 2} a_{\mathbf{k} 3}^{s 3} a_{\mathbf{k} 4}^{s 4}>>$ with various sets of superscripts. A part of these moments, for which the condition $s 1+s 2+s 4+s 4 \neq 0$ is met, can be put to zero, in accordance with definition (B.4). The rest forth moments are to be decoupled to the products of the second moments corresponding to the spectrum definition. By this way the first non-vanishing summands appear in the r.h.s. of the wavespectrum evolution equation (B.4), and they will be proportional to the second power in $S(\mathbf{k})$.

The described procedure can be continued by means of writing the proper equations for some of the uncoupled fourth moments, leading to appearance the fifth moments. After this, the procedure can be repeated for the fifth moments, and so on. Finally, this continuation ensure in the r.h.s. of the evolution equation (B.4) the fast converging series to powers in spectrum $S(\mathbf{k})$ (the reason of convergence is stated in section 3, ratio (3.5)). As far as the whole r.h.s. of the evolution equation (B.4) has the meaning of the dissipation mechanism (by origin of equation (4.17)), the solution described ensure the dissipation function $\operatorname{DIS}(S)$ in the following general kind

$$
\operatorname{DIS}(S, \mathbf{k}, \sigma)=\sum_{n=2}^{N} \alpha_{n}(\mathbf{k}, \sigma) F_{n}[S(\mathbf{k})] .
$$

Here, $F_{n}[S(\mathbf{k})]$ is the functional on the $n$-th power in the wave spectrum, and functions $\alpha_{n}(\ldots)$ are the implicit factors of dissipation intensity. Both multiplicands under sign of sum in expression (B.6) are written in the most general kind. Their specification is to be done on principals not related to the point of equation (4.17) solution (alike the similarity method).

The only specification can be done here, consisting in the local-type representation of functional $F_{n}[S(\mathbf{k})]$ via powers of $S(\mathbf{k})$. That is provided by the hypothesis (4.16) of nonresonant feature for the interactions considered. After this specification we have fully the same form of function $D I S(S)$, which was used as the initial representation for $D I S(S)$ in the similarity method (formulas (3.3), (3.4)). Thus, the theoretical justification of the latter is done.

\section{REFERENCES}

[1] Hasselmann, K., "On the spectral dissipation of ocean wave due white capping", Boundary Layer Meteorol., v. 6, pp. 107-127, 1974.

[2] Komen, G. L., L. Cavaleri, M. Donelan et al., Dynamics and Modelling of Ocean Waves, Cambridge University Press, UK, 1994.

[3] Cavaleri, L. et al. (The WISE group), "Wave modelling - the state of the art", Progress in oceanography,v.75, pp. 603-674, 2007.

[4] Polnikov, V. G., "Wind-Wave Model with an Optimized Source Function (English transl.)", Izvestiya, Atmospheric and Oceanic Physic, v. 41, pp. 594-610, 2005.

[5] Longuet-Higgins, M.S., "On wave breaking and the equilibrium spectrum of wind generated waves". Proc. Roy. Soc., v. A310, pp. 151-159, 1969.

[6] Battjes, J.A., and J.P.F.M. Janssen, "Energy Loss and Set-up due to Breaking of Random Waves", Proc. Coastal Eng. Conf., 16th, pp. 569-587, 1978.

[7] Phillips, O.M., "Spectral and statistical properties of the equilibrium range in wind-generated gravity waves" J. Fluid Mech., v. 156, pp. 505-631, 1985.

[8] Efimov, V. V., and V. G. Polnikov, Numerical modelling of wind waves (in Russian). Naukova dumka Publishing house. Kiev, 1991.

[9] Zaslavskii, M.M.," The blocking spectra for wind waves (in Russian)", Izvestiya, Atmospheric and Oceanic Phys., v. 35, pp. 269-277, 1999.

[10] Donelan, M. A., "A nonlinear dissipation function due to wave breaking”, in Proc. ECMWF Workshop on Ocean Wave Forecasting, Reading, United Kingdom, ECMWF, pp. 87-94, 2001.

[11] Chalikov D., D. Sheinin,, "Direct Modeling of One- dimensional Nonlinear Potential Waves", Nonlinear Ocean Waves, ed. Perrie W. Advances in Fluid Mechanics, v. 17, pp. 207 - 
$222,1998$.

[12] Zakharov, V.E., A.O. Korotkevich, A. Pushkarev, and D. Resio, "Coexistence of Weak and Strong Wave Turbulence in a Swell Propagation", Phys. Rev. Lett., v. 99, pp. 16-21, 2007

[13] Banner, M.L., and I.R. Young, "Modeling spectral dissipation in the evolution of wind waves. Part I: Assessment of existing model performance", J. Phys. Oceanogr., v. 24, pp. 1550 $-1571,1994$.

[14] Banner, M. L., and X. Tian, "On the determination of the onset of wave breaking for modulating surface gravity water waves", J. Fluid Mech., v. 367, pp. 107-137, 1998.

[15] Babanin, V.A., I.R. Young, and M.L. Banner, "Breaking probabilities for dominant surface waves on water of finite constant depth", J. Geophys. Res., v. 106C, pp. 11659-11676, 2001.

[16] Young, I. R., and A. V. Babanin, "Spectral distribution of energy dissipation of wind-generated waves due to dominant wave breaking", J. Phys. Oceanogr., v. 36, pp. 376-394, 2006.

[17] Babanin, A.V., and A.J. Van der Westhuysen, "Physics of "saturation-based" dissipation functions proposed for wave forecast models", J. Phys. Oceanogr., v. 38, pp. 1831-1841, 2008.

[18] Babanin, A. V., "Breaking of ocean surface waves", Acta Physica Slovaca, v. 59, pp. 305-553, 2009.

[19] Gemmrich, J.R., M.L. Banner, and C. Garret, "Spectrally Resolved Energy Dissipation Rate and Momentum Flux of Breaking Waves", J. Phys. Oceanogr., v. 39, pp. 1296-1312, 2009.

[20] Gemmrich, J,R., "Strong Turbulence in the Wave Crest Region”, J. Phys. Oceanogr., v. 40, pp. 583-595, 2010.

[21] Thomson, J., J.R. Gemmrich, and A.T. Jessup, "Energy dissipation and the spectral distribution of whitecaps", Geophysical Research Letters, v. 36, L11601, 2009.

[22] The WAMDI Group, "The WAM - a third generation ocean wave prediction model”, J. Phys. Oceanogr.,v.18, pp. 1775 $-1810,1988$.

[23] Tolman, H. L., and D. V. Chalikov, "Source terms in a third generation wind wave model", J. Phys. Oceanogr., v. 26, pp. 2497-2518, 1996.

[24] Yongzeng, Y., F. Qiao, W. Zhao, Y. Teng, and Y. Yuan, "MASNUM ocean wave numerical model in spherical coordinates and its application" (in Chinese), Acta Oceanologica Sinica, v. 27, pp. 1-7, 2005.

[25] Ardhuin, F., et. al, "Semiempirical Dissipation Source Functions for Ocean Waves. Part I: Definition, Calibration, and Validation”, J. Phys. Oceanogr., v. 40, pp. 1917 -1941, 2010.

[26] Hasselmann, K., "Grundglichungen der Seegangsvoruassage" (in German), Shifttechnik, v. 7, pp. 191-195, 1960.

[27] Efimov, V. V., and V. G. Polnikov, "Numerical simulations based on a wind wave model with the turbulent dissipation term” (in Russian), Marine Hydrophysical Journal, \#2, pp.
37-45, 1986.

[28] Polnikov, V. G., "On a description of a wind-wave energy dissipation function", in Proc. of conf. The Air-Sea Interface: Radio and Acoustic Sensing, Turbulence and Wave Dynamics, ed. by M. A. Donelan,,W. H. Hui, and W. J. Plant, Eds., Rosenstiel School of Marine and Atmospheric Science, University of Miami, 277-282, 1994.

[29] Polnikov, V. G., The study of nonlinear interactions in a wind wave spectrum (in Russian), Doctor of Science dissertation, Marine Hydrophysical Institute of NASU, Sebastopol, 1995.

[30] Polnikov, V. G., V. I. Dymov, T. A. Pasechnik, et al., "Testing and Verifying the Wind Wave Model with an Optimized Source Function (English transl.)", Oceanology, v. 48, pp. 7-14, 2008.

[31] Polnikov, V.G., and V. Innocentini, "Comparative study performance of wind wave model: WAVEWATCH- modified by the new source function", Engineering Applications of Computational Fluid Mechanics, v. 2, pp. 466-481, 2008.

[32] Babanin, A.V., and B.K. Haus, "On the Existence of Water Turbulence Induced by Nonbreaking Surface Waves", J. Phys. Oceanogr., v. pp. 2675-2679, 2009.

[33] Dai, D., F. Qiao, W. Sulisz, L. Han, and A. Babanin, “An Experiment on the Nonbreaking Surface-Wave-Induced Vertical Mixing", J. Phys. Oceanogr., v. 40, pp. 2180-2188, 2010.

[34] Polnikov, V.G., "An Extended Verification Technique for Solving Problems of Numerical Modeling of Wind Waves (English transl.)", Izvestiya, Atmospheric and Oceanic Physics, v. 46, pp. 551-564, 2010.

[35] Monin, A. S., A.M. Yaglom, Statistical Fluid Mechanics: Mechanics of Turbulence, v. 1. The MIT Press, Cambridge, Massachusets, and London, England, 1971.

[36] Phillips, O.M., "The equilibrium range in the spectrum of wind-generated waves", J. Fluid Mech., v. 4, pp. 231-245, 1958.

[37] Toba, Y., "Local balance in the air-sea boundary processes. Pt. 1: On the growth process of wind waves", J. Oceanogr. Soc. Japan, v. 28, pp. 109-121, 1972.

[38] Zakharov, V. E., "Hamiltonian formalism in the theory of waves in nonlinear media with dispersion (in Russian)", Izv. VUZov, Radiofizika, v. 17, pp. 431-453, 1974.

[39] Polnikov, V.G., Nonlinear theory for a stochastic wave field in water (in Russian), LENAND publishing house. Moscow, Russia, 2007.

[40] Polnikov, V. G., "The Role of Wind Waves in Dynamics of the Air-Sea Interface" (English transl.), Izvestiya, Atmospheric and Oceanic Physics, v. 45, pp. 346-356, 2009.

[41] Krasitskii, V. P., "On reduced equations in the Hamiltonian theory of weakly nonlinear surface waves", J. Fluid Mech., v. 272, pp. 1-20, 1994.

[42] Babanin A, et al, "Numerical investigation of spectral evolution of wind wave. Part II. Dissipation term and evolution", J. Phys. Oceanogr., v40, pp. 667-683, 2010. 